九州大学学術情報リポジトリ

Kyushu University Institutional Repository

\title{
Finite Element Simulation of Hydrogen Dispersion
}

\section{Kanayama, Hiroshi}

Department of Intelligent Machinery and Systems, Faculty of Engineering, Kyushu University

Maeda, kengo

Graduate School of Engineering, Kyushu University

Mino, Masayuki

Graduate School of Engineering, Kyushu University

Matsuura, Kazuo

Department of Intelligent Machinery and Systems, Faculty of Engineering, Kyushu University

http://hdl. handle. net/2324/5652

出版情報: Computational Fluid Dynamics Journal. 15 (1)，pp.101-106，2006-04. International Society of Computational Fluid Dynamics

バージョン :

権利関係 : 


\title{
FINITE ELEMENT SIMULATION OF HYDROGEN DISPERSION
}

\author{
Hiroshi KANAYAMA ${ }^{\dagger}$ Kengo MAEDA ${ }^{\ddagger}$ \\ Masayuki MINO ${ }^{\ddagger}$ Kazuo MATSUURA ${ }^{\natural}$
}

\begin{abstract}
Hydrogen is expected as new fuel instead of fossil fuel. It will be used as fuel of a fuel cell for which development is performed actively. Many scientists are studying characteristic features of hydrogen. But it is difficult to experiment the hydrogen dispersion in case of hydrogen leaks, because hydrogen has the properties that energy for ignition is lower, rate of flame propagation in air is faster and quenching distance is shorter than hydrocarbon fuel. Therefore clarifying the hydrogen dispersion with numerical analysis becomes important. Furthermore, hydrogen dispersion under various conditions can be clarified with numerical analysis, which is useful to use hydrogen safely. This paper deals with computer simulation of the hydrogen dispersion by a finite element method. The mathematical model of hydrogen dispersion is governed by the momentum equations, the continuity equation and the hydrogen mass conservation equation. The model presented here is a three-dimensional, incompressible, non-stationary model. This paper describes a finite element method with the stabilization technique for solving Navier-Stokes equations and the advection-diffusion equation for hydrogen concentration like the Boussinesq approximation of thermal convection problems. We use Bercovier-Pironneau elements for the velocity and the pressure, and smaller P1 elements for the concentration of hydrogen. A suitable implicit time difference is also used. Numerical results are shown for a sample model.
\end{abstract}

Key Words: Hydrogen, Dispersion, Finite element method, Stabilized method

\section{Introduction}

While various new energies are considered instead of fossile fuel, hydrogen is attracting the most interest as a leading energy in this century. It is greatly expanding the possibility from the use as a current industrial gas to the basic energy of a social system. It will be used as fuel of a fuel cell for which development is performed actively. A fuel cell immediately changes the chemical energy of hydrogen and oxygen to electrical

Received on April 5, 2006.

$\dagger$ Kyushu University, 744 Motooka, Nishi-ku, Fukuoka, Professor, Department of Intelligent Machinery and Systems, Faculty of Engineering, kanayama@mech.kyushuu.ac.jp

$\ddagger$ Kyushu University, 744 Motooka, Nishi-ku, Fukuoka, Graduate Student, Graduate School of Engineering

$\natural$ Kyushu University, 744 Motooka, Nishi-ku, Fukuoka, Research Scientist, Department of Intelligent Machinery and Systems, Faculty of Engineering

(C)Copyright: Computational Fluid Dynamics JOURNAL 2002 energy. This conversion has high efficiency and low emissions. In the demand for the zero emission and the $\mathrm{CO}_{2}$ reduction, the expectation for clean hydrogen is growing more and more.

Many scientists are studying characteristic features of hydrogen. But it is difficult to experiment the hydrogen dispersion in case of hydrogen leaks, because hydrogen has a high diffusion coefficient and low kinematic viscosity. Hence clarifying the hydrogen dispersion with numerical analysis becomes important.

This paper demonstrates a simulation of the hydrogen dispersion by a finite element method with the stabilization technique [1]. The organization of the paper is as follows. In section 2, a three-dimensional, incompressible, non-stationary model is developed to simulate the hydrogen dispersion. The equations consist of the momentum equations, the continuity equation and the hyrogen mass conservation equation. Section 3 describes a finite elment method with the stabilization technique for solving Navier-Stokes equations and the advection-diffusion equation for hydrogen concentration like the Boussinesq approximation of thermal convection problems [2]. We use Bercovier-Pironneau ele- 
ments [3] for the velocity and the pressure, and smaller P1 elements for the concentration of hydrogen. The backward Euler method is used for time integration. In Section 4, numerical results are reported. Finally, conclusions are described in Section 5.

\section{A Mathematical Model}

A three-dimensional incompressible non-stationary model of hydrogen dispersion is developed to simulate the hydrodynamics of gas flow. Fig. 1 shows a computational model of a hallway [4]. The hydrogen leaks from the floor at the left end of the hallway. There are a roof vent and a lower door vent for the gas ventilation at the right end of the hallway.

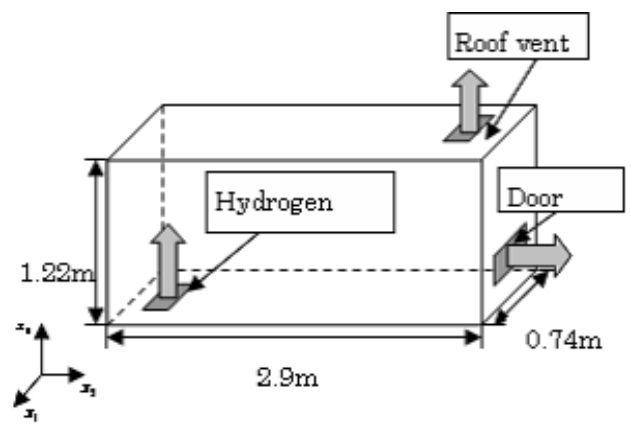

Fig.1: The ventilation model

\subsection{Basic Equations}

The conservation equations of momentum and mass are as follows:

$$
\begin{gathered}
\frac{\partial \mathbf{u}}{\partial t}+(\mathbf{u} \cdot \nabla) \mathbf{u}-2 \nu \nabla \cdot D(\mathbf{u})+\nabla p=-\beta C \mathbf{g}, \\
\nabla \cdot \mathbf{u}=0 .
\end{gathered}
$$

The mass conservation equation for the hydrogen is written as:

$$
\frac{\partial C}{\partial t}+\mathbf{u} \cdot \nabla C-a \triangle C=S .
$$

In the above, $\mathbf{u}=\left(u_{1}, u_{2}, u_{3}\right)^{T}$ is the velocity $[\mathrm{m} / \mathrm{s}]$; $t$ is time $[s] ; \nu$ is the kinematic viscosity coefficient $\left[\mathrm{m}^{2} / \mathrm{s}\right] ; p$ is the mixture gas pressure normalized by the density $\left[\mathrm{m}^{2} / \mathrm{s}^{2}\right] ; \mathbf{g}=\left(g_{1}, g_{2}, g_{3}\right)^{T}$ is the gravity $\left[\mathrm{m} / \mathrm{s}^{2}\right] ; \beta$ is the coefficient $[-] ; C$ is the mass concentration of hydrogen [mass\%] $a$ is the hydrogen diffusion coefficient in air $\left[\mathrm{m}^{2} / \mathrm{s}\right] ; S$ is the source term $[1 / s]$; and $D_{i j}$ is the rate of strain tensor $[1 / s]$ defined by

$$
D(\mathbf{u}) \equiv \frac{1}{2}\left(\frac{\partial u_{i}}{\partial x_{j}}+\frac{\partial u_{j}}{\partial x_{i}}\right), \quad i, j=1,2,3 .
$$

\subsection{Boundary Conditions}

Here, the boundary conditions are described precisely. $\Gamma_{\text {inlet }}, \Gamma_{\text {roof }}, \Gamma_{\text {door }}$ denote the boundary of the hydrogen inlet, the boundary of the root vent, and the boundary of the door vent, respectively.

At the inlet, the hydrogen leaks in the vertical direction. The velocity and the concentration are specified as follows;

$$
\left\{\begin{array}{l}
u_{1}=u_{2}=0 \quad[\mathrm{~m} / \mathrm{s}] \\
u_{3}=0.02 \quad[\mathrm{~m} / \mathrm{s}], \quad \text { on } \Gamma_{\text {inlet }} . \\
C=100 \quad[\text { mass } \%]
\end{array}\right.
$$

Boundary conditions of both vents are the following, because at the roof vent and the door vent, the hydrogen is discharged outside freely.

$$
\left\{\begin{array}{l}
\sum_{j=1}^{3} \sigma_{i j} n_{j}=0 \quad\left[\mathrm{~m}^{2} / \mathrm{s}^{2}\right], \\
C=0 \quad[\text { mass } \%]
\end{array}\right.
$$

where $\sigma(\mathbf{u}, p)$ is the stress tensor normalized by the density $\left[\mathrm{m}^{2} / \mathrm{s}^{2}\right]$ defined by

$$
\sigma_{i j}=-p \delta_{i j}+2 \nu D_{i j}(\mathbf{u}), \quad i, j=1,2,3,
$$

with the Kronecker delta $\delta_{i j}$.

At the other boundary, there is no inflow of hydrogen.

$\left\{\begin{array}{l}u_{1}=u_{2}=u_{3}=0 \quad[\mathrm{~m} / \mathrm{s}], \\ a \frac{\partial C}{\partial n}=0 \quad[\mathrm{~m} / \mathrm{s}],\end{array} \quad\right.$ on $\quad \partial \Omega-\left(\Gamma_{\text {inlet }}+\Gamma_{\text {roof }}+\Gamma_{\text {door }}\right)$,

where $n$ is the unit normal vector.

\subsection{Initial Conditions}

The initial conditions are described as follows;

$$
\left\{\begin{array}{l}
u_{1}=u_{2}=u_{3}=0 \quad[\mathrm{~m} / \mathrm{s}], \quad \text { in } \Omega \\
C=0 \quad[\text { mass } \%]
\end{array}\right.
$$

\section{Numerical Method}

\subsection{Formulation}

Let $\Omega$ be a three-dimensional polyhedral domain with the boundary $\partial \Omega . \quad \Gamma_{u}$ denotes the boundary with specifed velocity. $\Gamma_{c}$ denotes the boundary with specified concentration. We consider the non-stationary Navier-Stokes equations and the advection-diffusion equation as follows;

$\frac{\partial \mathbf{u}}{\partial t}+(\mathbf{u} \cdot \nabla) \mathbf{u}-2 \nu \nabla \cdot D(\mathbf{u})+\nabla p=-\beta C \mathbf{g} \quad$ in $\quad \Omega \times(0, T)$, 


$$
\begin{array}{r}
\nabla \cdot \mathbf{u}=0 \quad \text { in } \quad \Omega \times(0, T), \\
\frac{\partial C}{\partial t}+\mathbf{u} \cdot \nabla C-a \triangle C=S \quad \text { in } \quad \Omega \times(0, T), \\
\mathbf{u}=\widehat{\mathbf{u}} \quad \text { on } \quad \Gamma_{u} \times(0, T), \\
C=\widehat{C} \quad \text { on } \quad \Gamma_{c} \times(0, T), \\
\sum_{j=1}^{3} \sigma_{i j} n_{j}=0 \quad \text { on } \quad\left(\partial \Omega-\Gamma_{u}\right) \times(0, T), \\
a \frac{\partial C}{\partial n}=0 \quad \text { on } \quad\left(\partial \Omega-\Gamma_{c}\right) \times(0, T), \\
\mathbf{u}=\mathbf{u}^{0}, \quad C=C^{0} \quad \text { in } \quad \Omega \quad \text { at } \quad t=0,
\end{array}
$$

where $T$ is the total time $[s] ; \mathbf{u}^{0}$ is the initial velocity $[\mathrm{m} / \mathrm{s}] ; C^{0}$ is the initial concentration [mass\%]; $\widehat{\mathbf{u}}$ is the boundary velocity $[\mathrm{m} / \mathrm{s}]$; and $\widehat{C}$ is the boundary concentration [mass\%].

As the weak form, the following system is considered;

$$
\begin{gathered}
\left(\frac{\partial \mathbf{u}}{\partial t}, \mathbf{v}\right)+((\mathbf{u} \cdot \nabla) \mathbf{u}, \mathbf{v})+(2 \nu D(\mathbf{u}), D(\mathbf{v})) \\
-(p, \nabla \cdot \mathbf{v})+(\beta C \mathbf{g}, \mathbf{v})=0 \quad \text { for } \quad \mathbf{v} \in V, \\
-(\nabla \cdot \mathbf{u}, q)=0 \quad \text { for } \quad q \in Q,
\end{gathered}
$$

$\left(\frac{\partial C}{\partial t}, \theta\right)+(\mathbf{u} \cdot \nabla C, \theta)+(a \nabla C, \nabla \theta)=(S, \theta) \quad$ for $\quad \theta \in \Theta$.

Here, $(\cdot, \cdot)$ denotes the $L^{2}$-inner product over $\Omega, L^{2}(\Omega)$ denotes the space of square summable functions in $\Omega$, and $H^{1}(\Omega)$ is the space of functions in $L^{2}(\Omega)$ with derivatives up to the first order. Then,

$$
\begin{gathered}
V\left(g_{1}\right) \equiv\left\{\mathbf{v} \in\left(H^{1}(\Omega)\right)^{3} ; \quad \mathbf{v}=g_{1} \quad \text { on } \quad \Gamma_{u}\right\} \\
V \equiv V(0) \\
Q \equiv\left\{q \in L^{2}(\Omega)\right\} \\
\Theta\left(g_{2}\right) \equiv\left\{\theta \in H^{1}(\Omega) ; \quad \theta=g_{2} \text { on } \Gamma_{c}\right\} \\
\Theta \equiv \Theta(0)
\end{gathered}
$$

\subsection{Finite Element Approximation}

Let us consider approximations of above formulations. The finite element method is used for discretization of space, and the backward Euler method is used for discretization of time. The Bercovier-Pironneau tetrahedral elements are used for approximations of velocity and pressure. The concentration is approximated like the velocity. The approximation scheme without stabilized terms is first explained as follows;

$$
\begin{aligned}
& \left(\frac{\mathbf{u}_{h}^{(n+1)}-\mathbf{u}_{h}^{(n)}}{\Delta t}, \mathbf{v}_{h}\right)+\left(\left(\mathbf{u}_{h}^{(n)} \cdot \nabla\right) \mathbf{u}_{h}^{(n+1)}, \mathbf{v}_{h}\right) \\
& +\left(2 \nu D\left(\mathbf{u}_{h}^{(n+1)}\right), D\left(\mathbf{v}_{h}\right)\right)-\left(p_{h}^{(n+1)}, \nabla \cdot \mathbf{v}_{h}\right) \\
& \quad+\left(\beta C_{h}^{(n+1)} \mathbf{g}, \mathbf{v}_{h}\right)-\left(\nabla \cdot \mathbf{u}_{h}^{(n+1)}, q_{h}\right) \\
& +\left(\frac{C_{h}^{(n+1)}-C_{h}^{(n)}}{\Delta t}, \theta_{h}\right)+\left(\mathbf{u}_{h}^{(n)} \cdot \nabla C_{h}^{(n+1)}, \theta_{h}\right) \\
& +\left(a \nabla C_{h}^{(n+1)}, \nabla \theta_{h}\right)=\left(S, \theta_{h}\right)
\end{aligned}
$$$$
\text { for } \quad \mathbf{v}_{h} \in V_{h}, \quad q_{h} \in Q_{h}, \quad \theta_{h} \in \Theta_{h},
$$

where $\Delta t$ denotes a time increment. $\mathbf{u}_{h}^{(n)}, \quad p_{h}^{(n)}$ and $C_{h}^{(n)}$ denote the finite element approximations of $\mathbf{u}, p$ and $C$ at time $n \Delta t$, respectively.

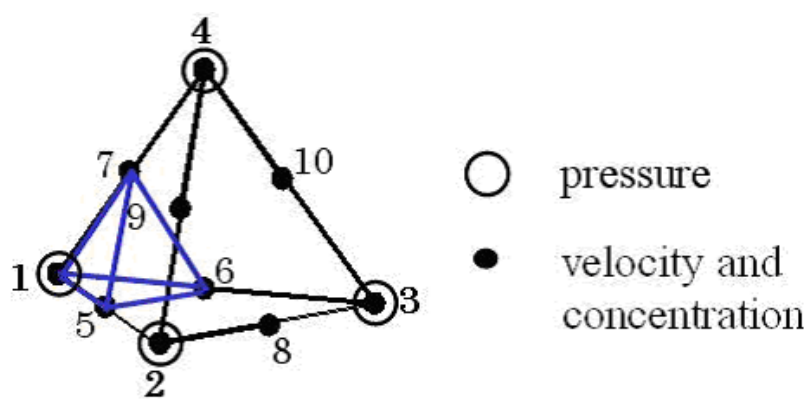

Fig.2: Bercovier-Pironneau elements

\subsection{Stabilized Methods}

Now, our computational scheme with stabilized terms is introduced as follows;

$$
\begin{gathered}
\left(\frac{\mathbf{u}_{h}^{(n+1)}-\mathbf{u}_{h}^{(n)}}{\Delta t}, \mathbf{v}_{h}\right)+\left(\left(\mathbf{u}_{h}^{(n)} \cdot \nabla\right) \mathbf{u}_{h}^{(n+1)}, \mathbf{v}_{h}\right) \\
+\left(2 \nu D\left(\mathbf{u}_{h}^{(n+1)}\right), D\left(\mathbf{v}_{h}\right)\right)-\left(p_{h}^{(n+1)}, \nabla \cdot \mathbf{v}_{h}\right)+\left(\beta C_{h}^{(n+1)} \mathbf{g}, \mathbf{v}_{h}\right) \\
+\sum_{K \in \Im_{h / 2}} \tau_{K}^{N S}\left(\begin{array}{l}
\left(\mathbf{u}^{(n+1)}-\mathbf{u}^{(n)}\right) / \Delta t \\
+\left(\mathbf{u}^{(n)} \cdot \nabla\right) \mathbf{u}^{(n+1)} \\
+\nabla p^{(n+1)}+\beta C^{(n+1)} \mathbf{g}, \\
\left(\mathbf{u}^{(n)} \cdot \nabla\right) \mathbf{v}-\nabla q
\end{array}\right) \\
-\left(\nabla \cdot \mathbf{u}_{h}^{(n+1)}, q_{h}\right)+\sum_{K \in \Im_{h / 2} \tau_{K}^{C O}\left(\nabla \cdot \mathbf{u}_{h}^{(n+1)}, \nabla \cdot \mathbf{v}_{h}\right)_{K}+} \\
\left(\begin{array}{l}
\left.C_{h}^{(n+1)}-C_{h}^{(n)}, \theta_{h}\right)+\left(\mathbf{u}_{h}^{(n)} \cdot \nabla C_{h}^{(n+1)}, \theta_{h}\right)+\left(\kappa \nabla C_{h}^{(n+1)}, \nabla \theta_{h}\right) \\
\Delta t
\end{array}\right. \\
\sum_{K \in \Im_{h / 2}} \tau_{K}^{A D}\left(\begin{array}{l}
\left(C_{h}^{(n+1)}-C_{h}^{(n)}\right) / \Delta t \\
+\mathbf{u}^{(n)} \cdot \nabla C^{(n+1)}, \\
\mathbf{u}_{h}^{(n)} \cdot \nabla \theta_{h}
\end{array}\right)_{K}=
\end{gathered}
$$




$$
\left(S, \theta_{h}\right)+\sum_{K \in \Im_{h / 2}} \tau_{K}^{A D}\left(S, \mathbf{u}_{h}^{(n)} \cdot \nabla \theta_{h}\right)_{K},
$$

where $(\cdot, \cdot)_{K}$ denotes the $L^{2}$-inner product over $\mathrm{K}$. The stabilized parameter $\tau_{K}^{N S}, \tau_{K}^{C O}$ and $\tau_{K}^{A D}$ are defined by

$$
\begin{gathered}
\tau_{K}^{N S} \equiv \min \left\{\frac{\Delta t}{2}, \frac{h_{K}}{2\left|\mathbf{u}_{h}^{(n)}\right|_{\infty}}, \frac{h_{K}^{2}}{24 \nu}\right\}, \\
\tau_{K}^{C O} \equiv \min \left\{\frac{\lambda\left|\mathbf{u}_{h}^{(n)}\right|_{\infty}^{2} h_{K}^{2}}{12 \nu}, \lambda\left|\mathbf{u}_{h}^{(n)}\right|_{\infty} h_{K}\right\}, \\
\tau_{K}^{A D} \equiv \min \left\{\frac{\Delta t}{2}, \frac{h_{K}}{2\left|\mathbf{u}_{h}^{(n)}\right|_{\infty}}, \frac{h_{K}^{2}}{12 a}\right\} .
\end{gathered}
$$

where the constant $\lambda$ is set to be $1,|\mathbf{u}|_{\infty}$ denotes the maximum norm of $\mathbf{u}$ in $K$, and $h_{K}$ denotes the diameter of $K$. The symbol $\Im_{h / 2}$ denotes the subdivision of the triangulation $\Im_{h}$, which is constructed using eight small tetrahedral pieces of each element of $\Im_{h}$.

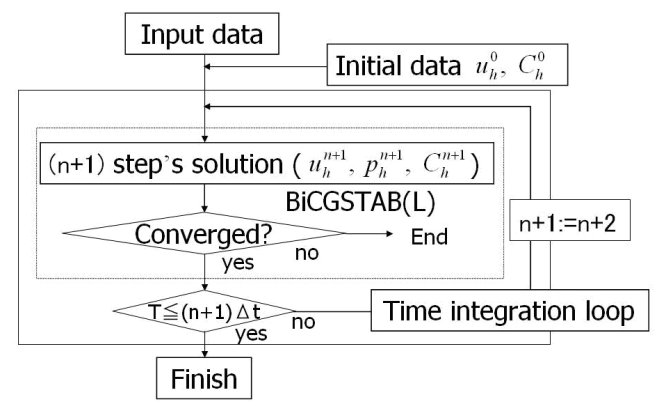

Fig.3: Analysis flow chart

\section{Numerical Results}

\subsection{Parameters}

\begin{tabular}{|c|c|}
\hline Parameters & Values \\
\hline kinematic viscosity & $1.05 \times 10^{-4} \quad\left[\mathrm{~m}^{2} / \mathrm{s}\right]$ \\
\hline diffusion coefficient in air & $6.1 \times 10^{-5} \quad\left[\mathrm{~m}^{2} / \mathrm{s}\right]$ \\
\hline the coefficient $\beta$ & $13.4[-]$ \\
\hline gravity & $(0,0,-9.8) \quad\left[m / s^{2}\right]$ \\
\hline the source term $\mathrm{S}$ & $0 \quad[1 / s]$ \\
\hline
\end{tabular}

Several parameters including gas properties are summarized in Table 1. Related sizes of the hallway are

Table 1: Parameters

shown in Table 2.

\subsection{The Coefficient $\beta$}

In the thermal convection problems, the coefficient $\beta$ is known as the thermal expansion coefficient. But, in
Table 2: Related sizes of the hallway

\begin{tabular}{|c|c|c|c|}
\hline- & $x_{1}(m)$ & $x_{2}(m)$ & $x_{3}(m)$ \\
\hline inlet size & 0.3 & 0.15 & 0.00 \\
\hline roof vent size & 0.30 & 0.15 & 0.00 \\
\hline door vent size & 0.30 & 0.00 & 0.15 \\
\hline
\end{tabular}

this hydrogen dispersion, the coefficient $\beta$ is unknown. We determine the coefficient $\beta$ by the following; The buoyancy force is represented by;

$$
\mathbf{f}=\left(1-\frac{\rho_{a i r}}{\rho}\right) \mathbf{g}
$$

where $\mathbf{f}$ is the buoyancy force normalized by the density $\left[\mathrm{m} / \mathrm{s}^{2}\right] ; \rho_{\text {air }}$ is the density of air $\left[\mathrm{kg} / \mathrm{m}^{3}\right] ; \rho$ is the mixture gas density $\left[\mathrm{kg} / \mathrm{m}^{3}\right]$ represented by;

$$
\rho=\frac{P_{m}}{\left[C R_{H_{2}}+(1-C) R_{a i r}\right] T_{m}},
$$

where $P_{m}$ is the pressure of the mixture gas $[\mathrm{Pa}] ; T_{m}$ is the absolute temperature $[K] ; R_{H_{2}}$ is the gas constant of hydrogen $[\mathrm{J} /(\mathrm{kg} \cdot \mathrm{K})]$ and $R_{\text {air }}$ is the gas constant of air $[\mathrm{J} /(\mathrm{kg} \cdot \mathrm{K})]$. On the other hand, the buoyancy force in the Boussinesq approximation is represented by the following;

$$
\mathbf{f}=-\beta C \mathbf{g}
$$

We write Fig. 4 using the following parameters in Table 3. Fig. 4 shows the coefficient $\beta=13.4$.

Table 3: Parameters at $1[\mathrm{~atm}]$ and $20\left[{ }^{0} \mathrm{C}\right]$

\begin{tabular}{|c|c|}
\hline Parameters & \multicolumn{2}{|c|}{ Values } \\
\hline$\rho_{\text {air }}$ & $1.209 \quad\left[\mathrm{~kg} / \mathrm{m}^{3}\right]$ \\
\hline$R_{H_{2}}$ & $4,122 \quad[\mathrm{~J} /(\mathrm{kg} \cdot \mathrm{K})]$ \\
\hline$R_{\text {air }}$ & $287 \quad[\mathrm{~J} /(\mathrm{kg} \cdot \mathrm{K})]$ \\
\hline$T_{m}$ & $297 \quad[\mathrm{~K}]$ \\
\hline$P_{m}$ & $1.01 \times 10^{5}[\mathrm{~Pa}]$ \\
\hline
\end{tabular}

\subsection{Other Numerical Conditions}

The number of elements and degrees of freedom are 149,863 and 797,868 , respectively. A time increment sets $0.02[s]$. BiCGSTAB $(\mathbf{L})$ method [5] with the incomplete LDU factorization preconditioner is used as the solver for each step in the time integration. The number $\mathbf{L}$ is set to be 4 . The acceleration factor is set to be 1.05. Computation of the model was performed on a Pentium $4(3.0 \mathrm{GHz})$ with $1 \mathrm{CPU}$. It took about 45 hours for 450 time steps . The average number 


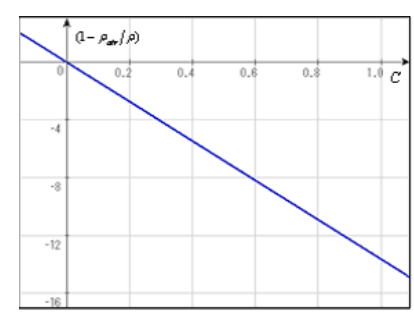

Fig.4: Relation between $C$ and $\left(1-\rho_{\text {air }} / \rho\right)$

of the $\operatorname{BiCGSTAB}(\mathbf{L})$ iteration was 19. Convergence criteria for the iteration is that the relative residual is less than $1.0 \times 10^{-6}$.

\subsection{Results}

Fig. 6 to Fig. 14 show the hydrogen concentration distributions from $1.0[s]$ to $9.0[s]$. The concentration iso-surface shows 4.0 [vol.\%] hydrogen concentration which is the lower flammable limit of the mixture gas.

\section{Conclusions}

The dispersion phenomena of hydrogen are modeled using the analogy of thermal convection problems with the Boussinesq approximation. The flow of hydrogen is grasped by a stabilized finite element method. We have to perform accuracy verification of computational results. It remains the next problem.

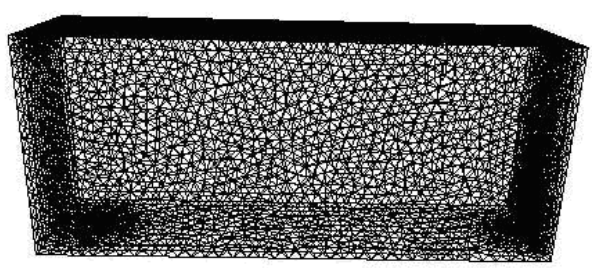

Fig.5: The finite element mesh

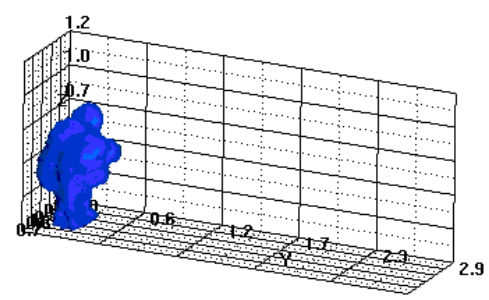

Fig.6: Concentration iso-surface of 4.0 [vol.\%] at 1.0 $[s]$

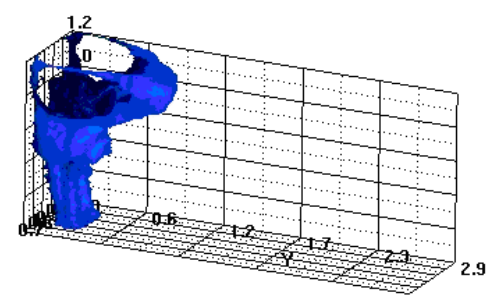

Fig.7: Concentration iso-surface of 4.0 [vol.\%] at 2.0 $[s]$

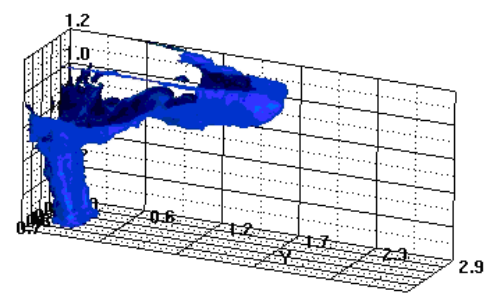

Fig.8: Concentration iso-surface of 4.0 [vol.\%] at 3.0 $[s]$

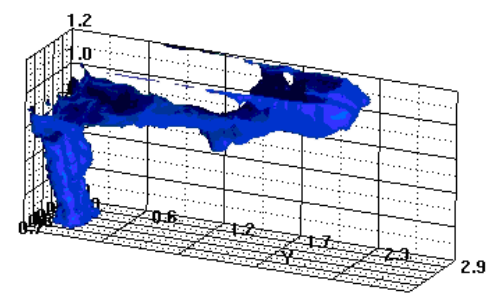

Fig.9: Concentration iso-surface of 4.0 [vol.\%] at 4.0 $[s]$

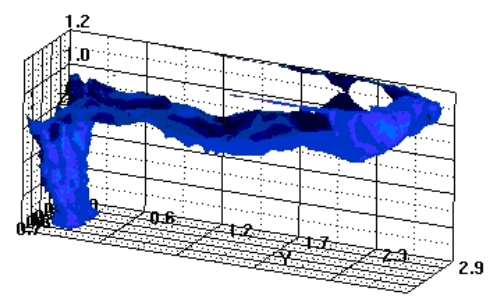

Fig.10: Concentration iso-surface of $4.0[$ vol.\%] at 5.0 $[s]$ 


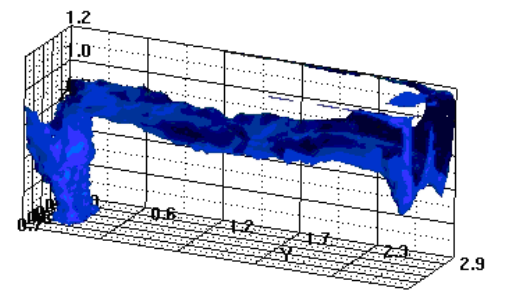

Fig.11: Concentration iso-surface of 4.0 [vol.\%] at 6.0 $[s]$

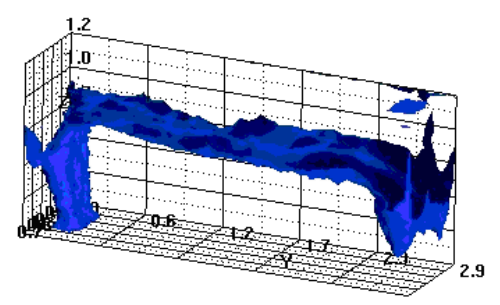

Fig.12: Concentration iso-surface of 4.0 [vol.\%] at 7.0 $[s]$

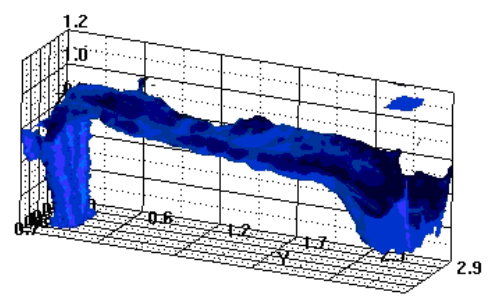

Fig.13: Concentration iso-surface of 4.0 [vol.\%] at 8.0

$[s]$

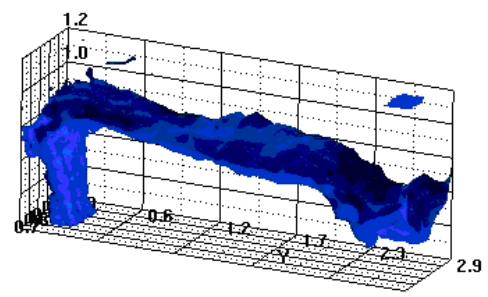

Fig.14: Concentration iso-surface of 4.0 [vol.\%] at 9.0 $[s]$

\section{REFERENCES}

[1] H. Kanayama, H. Kume and D. Tagami, Stabilized finite element method for stationary thermal convection equations, Proceedings of The Annual Meeting of The Japan Society for Industrial and Applied Mathematics, (2002) (in Japanese)

[2] D. Tagami and H. Itoh, A finite element analysis of thermal convection problems with the Joule heat, The Japan Journal of Industrial and Applied Mathematics, Vol. 20, No. 2, pp. 193-210, (2003)

[3] M. Bercovier and O. Pironneau, Error estimates for finite element method solution of the Stokes problem in the primitive variables, Numerische Mathematik, 33, pp. 211-224, (1979)

[4] V. Agarant, Z. Cheng and A. Tchouvelev, CFD modeling of hydrogen releases and dispersion in hydrogen energy station, Proceedings of The $15^{\text {th }}$ World Hydrogen Energy Conference, (2004)

[5] G. L. G. Sleijpen and D. R. Fokkema, $\operatorname{BiCGSTAB}(\mathbf{L})$ for linear equations involving unsymmetric matrices with complex spectrum, Electronic Transactions on Numerical Analysis, Vol. 1, pp. 11-32, (1993) 